\title{
Une lettre de Gotthold Ephraïm Lessing à sa mère
}

\author{
Jean-Louis Elloy
}

Traducteur : Jean-Louis Elloy

\section{(2) OpenEdition \\ Journals}

Édition électronique

URL : http://journals.openedition.org/rbnu/1538

DOI : $10.4000 /$ rbnu. 1538

ISSN : 2679-6104

\section{Éditeur}

Bibliothèque nationale et universitaire de Strasbourg

\section{Édition imprimée}

Date de publication : 1 mai 2016

Pagination : 82-87

ISBN : 9782859230623

ISSN : 2109-2761

\section{Référence électronique}

Jean-Louis Elloy, "Une lettre de Gotthold Ephraïm Lessing à sa mère », La Revue de la BNU [En ligne], 13 | 2016, mis en ligne le 01 mars 2020, consulté le 13 décembre 2020. URL : http:// journals.openedition.org/rbnu/1538; DOI : https://doi.org/10.4000/rbnu.1538

\section{cc) (i) (2)}

La Revue de la BNU est mise à disposition selon les termes de la Licence Creative Commons Attribution - Pas d'Utilisation Commerciale - Partage dans les Mêmes Conditions 4.0 International. 

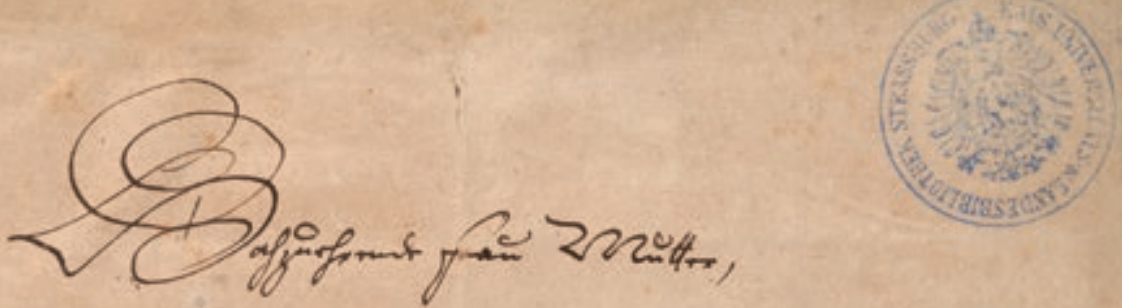

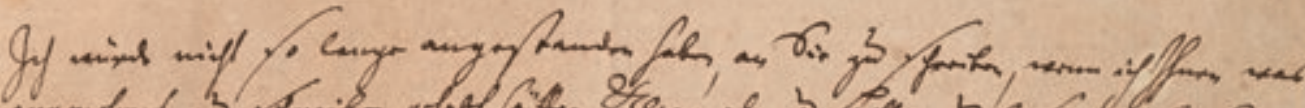

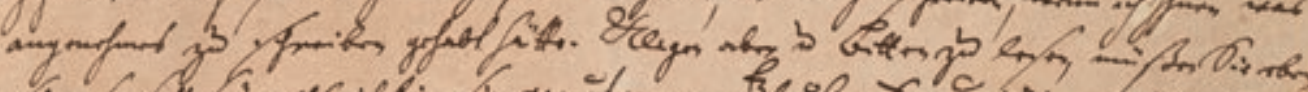

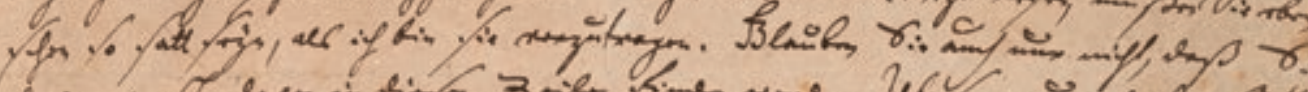

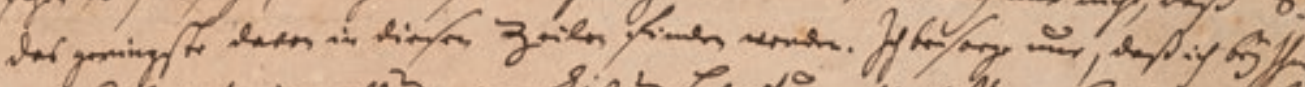

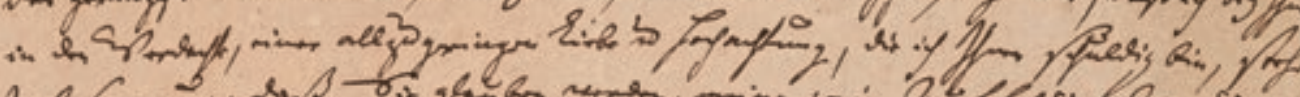

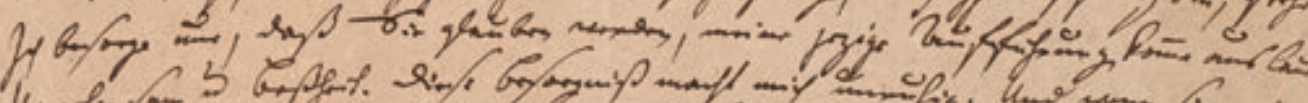

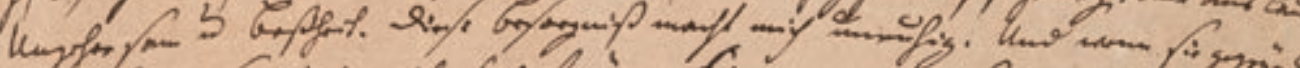

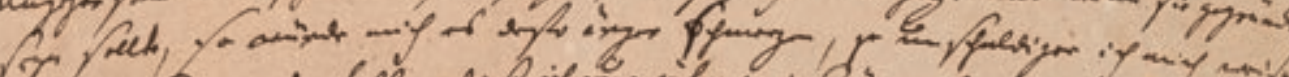

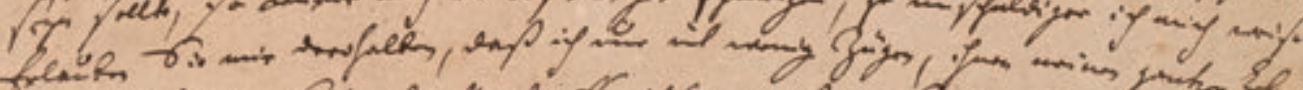

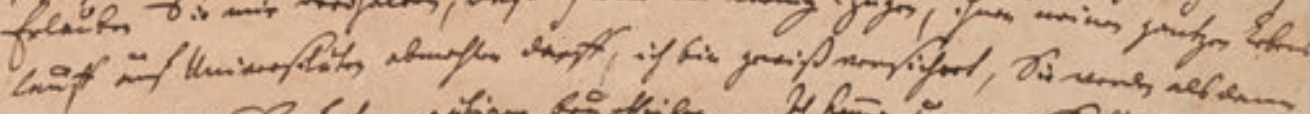

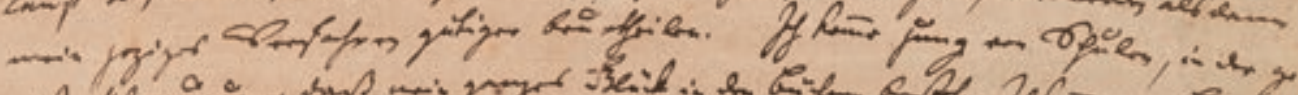

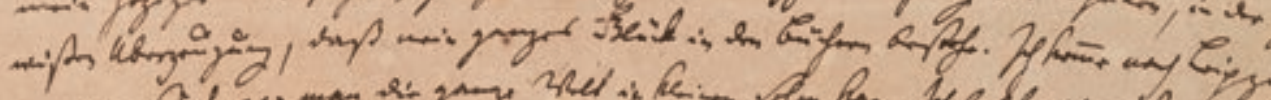

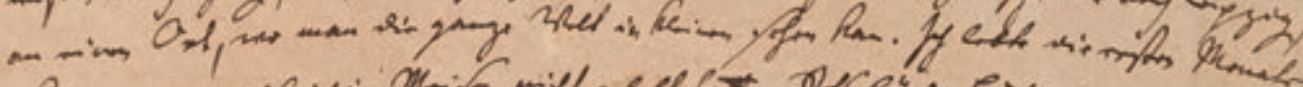

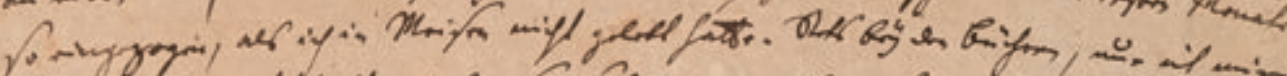

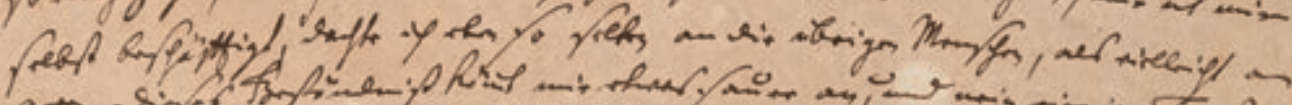

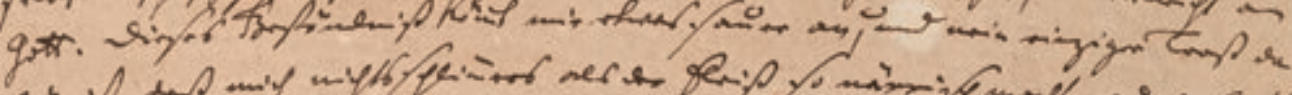

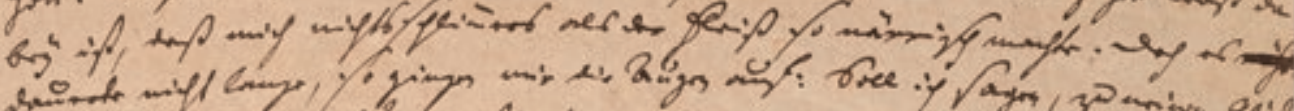

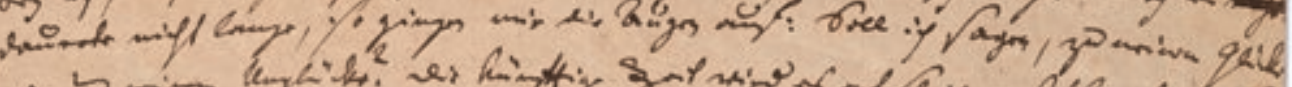

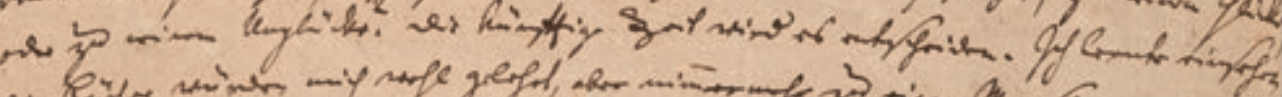

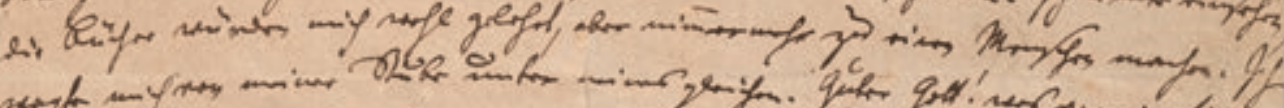

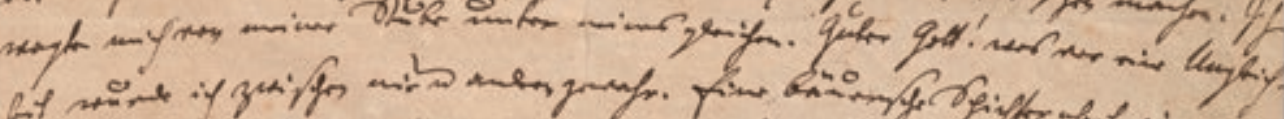

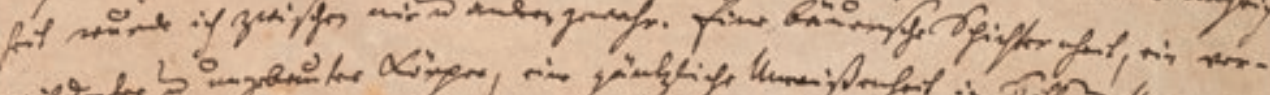

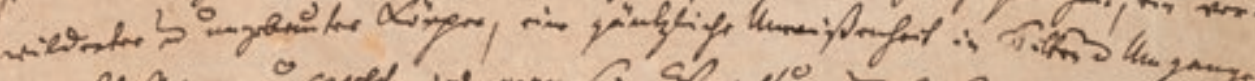

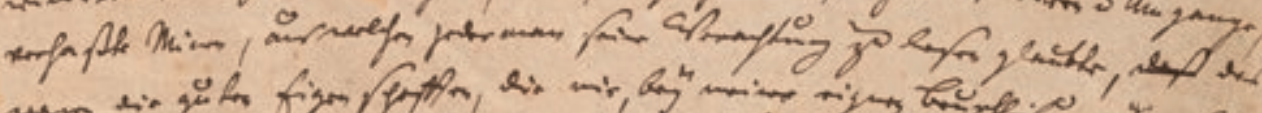

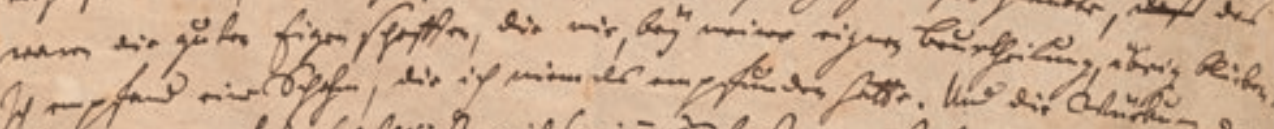

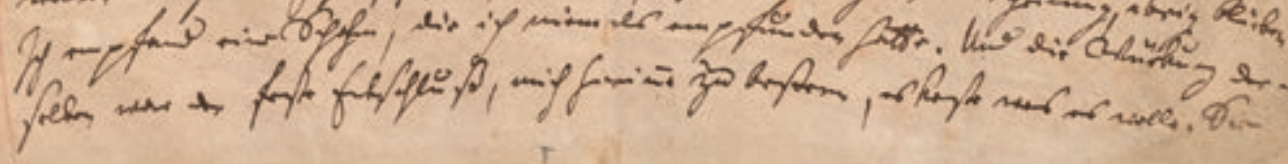




\section{UNE LETTRE DE GOTTHOLD EPHRAÏM LESSING À SA MÈRE}

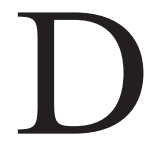

ans le numéro 12 de La Revue de la BNU, consacré à la thématique des dons et des legs, avait été présentée la bibliothèque de l'homme d'État autrichien Johann Philipp von Wessenberg-Ampringen, donnée à la Kaiserliche Universitäts- und Landesbibliothek zu Straßburg en 1876 . Cette riche collection comprenait, outre 3786 volumes, des manuscrits autographes de Johann Philipp lui-même, mais aussi de personnalités diverses, diplomates et hommes politiques de son temps en premier lieu... mais pas seulement : en effet, on y trouve aussi une lettre bien connue de tous les spécialistes de Lessing, dont l'importance pour comprendre la formation intellectuelle du dramaturge est capitale. Ce document, dont l'intérêt va bien au-delà du simple prestige de l'autographe, n'avait jusqu'ici jamais été traduit en français. Il nous a semblé qu'il y avait là l'occasion de réparer une lacune et de présenter aussi au public francophone un témoin capital de la maturation intellectuelle d'un auteur canonique du théâtre européen.

Comme beaucoup d'intellectuels de son époque, Gotthold Ephraïm Lessing (1729-1781), figure centrale de l'Aufklärung, est l'auteur d'une riche correspondance. Il est regrettable qu'elle n'ait été que très partiellement traduite en français car elle offre un ensemble documentaire très précieux pour suivre l'élaboration de ses théories littéraires et esthétiques. Les lettres les plus connues, adressées notamment à Moses Mendelssohn, Friedrich Nicolai ou Christian Friedrich Voss, brassent les thèmes essentiels de sa réflexion théorique sur le théâtre. Lessing écrit avant tout à des proches et amis et attend d'eux, comme il l'a lui-même répété dans ses lettres, qu'ils engagent avec lui un véritable débat cri- tique. De manière singulière, surtout au regard du style épistolaire contemporain, il ne se confie que très peu dans sa correspondance, et reste sobre dans l'expression de ses sentiments personnels. C'est pourquoi sa lettre à sa mère datée du 20 janvier 1749 est exceptionnelle à plus d'un titre.

Souvent citée par les biographes, elle nous apprend des informations intimes et précises sur l'état d'esprit de Lessing à un moment décisif de son parcours social et intellectuel. Elle prend donc la forme inhabituelle chez lui d'une confidence autobiographique, voire d'une confession. Pour autant, on y reconnaît discrètement mais sûrement ce que le dramaturge développe plus tard dans ses échanges épistolaires avec d'autres correspondants : une vision nouvelle du théâtre. Dans une lettre de 1773, Lessing déclare n'avoir jamais écrit dans sa correspondance une seule ligne qui ne puisse être lue aussi bien par le monde entier que par son destinataire particulier. Cela vaut aussi pour cette lettre de 1749, qui s'adresse certes à sa mère dans une situation d'urgence bien particulière, mais qui voit aussi au-delà et pose déjà quelques fondements de ce dialogue esthétique engagé avec ses contemporains.

En 1749, Lessing a vingt ans. Il séjourne alors à Berlin chez un ami, Christlob Mylius, journaliste, savant et écrivain, qu'il connaît de longue date et qui est, comme lui, originaire de Kamenz. C'est en effet dans cette ville saxonne, en Haute-Lusace, que Lessing est né et que réside sa famille depuis plusieurs générations. Mylius joue un rôle initiatique pour son ami et lui ouvre, grâce à ses relations, la voie du journalisme et du théâtre. Il est aussi au cœur du conflit qui l'oppose à ses parents. Ces derniers le considèrent comme un mécréant qui aurait dévoyé leur fils en le détournant de ses études 
universitaires. Le nom de Mylius n'apparaît pas dans la lettre mais c'est à lui que Lessing fait allusion lorsqu'il évoque la haine injustifiée de sa mère pour un homme qui l'aurait influencé.

Avant d'en arriver là, Lessing dresse un bilan de ses études. Le rappel de quelques faits permet de mieux suivre les allusions qui l'accompagnent. Élève remarqué pour ses dons et ses facilités, Lessing quitte assez vite l'école communale de Kamenz pour entrer dans le collège princier de Meissen, réservé aux élèves méritants qui peuvent dans ce cas bénéficier d'une bourse. La famille de Lessing, dont le père est pasteur principal à Kamenz, mène un train de vie modeste. Les parents auront douze enfants et ceux qui, parmi eux, en viendront à suivre des études ne pourront le faire qu'à l'aide de bourses, souvent dans des conditions difficiles. Ces soucis d'argent reviennent constamment dans la correspondance de Lessing avec ses parents. En 1746, il commence ses études universitaires à Leipzig et s'inscrit en théologie, sans doute sous l'influence paternelle. Mais il se consacre à des activités de journaliste et de dramaturge, publie ses premiers articles dans des revues éditées par Mylius et fréquente la troupe de Caroline Neuber, actrice renommée de cette époque.

Ses parents ont vent de ce qu'ils considèrent comme une dérive dangereuse. Ils le rappellent alors à Kamenz, en inventant même une maladie de la mère de Lessing pour le convaincre de faire le déplacement. Il lui faut attendre un trimestre, jusqu'en avril 1748 , pour pouvoir repartir à Leipzig après avoir promis de reprendre ses études. Mais là-bas ses dettes s'accumulent, sans doute parce qu'il s'était porté garant pour les emprunts engagés par la troupe de Neuber qui part précipitament à Vienne pour échapper à ses créanciers. Il doit lui-même fuir Leipzig, se rend tout d'abord à Wittenberg où il obtient une nouvelle bourse après s'être inscrit en faculté de médecine; mais dès novembre, une fois ses dettes acquittées, il gagne Berlin. Là, il poursuit très activement ses activités théâtrales, journalistiques et critiques, toujours avec le soutien et l'amitié d'éditeurs de revues comme Mylius - encore lui - ou Christian Friedrich Voss, et élargit toujours plus son cercle de connaissances. Ce parcours peut sembler chaotique. En réalité, Lessing le construit avec beaucoup plus de rigueur et d'autonomie qu'on ne pourrait le croire au vu de ces errances géographiques.

Précisément par ce qu'elle dit, parfois par ce qu'elle élude, cette lettre rend compte d'un fil conducteur. Dans ce bilan sur ses choix intellectuels, Lessing semble parfois, comme on l'a dit, s'adresser à ses contempo- rains autant qu'à sa mère. Il présente sa passion pour le théâtre et précisément pour la comédie comme principe unificateur de ses décisions. Bien évidemment, il n'établit pas de lien entre ses dettes et ses fréquentations théâtrales ; il se garde bien de préciser que s'il est prêt à quitter Berlin pour satisfaire les demandes de sa mère, c'est éventuellement pour rejoindre des gens de théâtre dans d'autres villes, notamment la troupe de Neuber qui a émigré à Vienne. Mais il n'hésite pas non plus à justifier son œuvre de dramaturge, avec une fierté affichée lorsqu'il fait part, dans un passage central de la lettre, de son souhait de rivaliser avec les grands modèles étrangers. Les quelques phrases sur l'édification morale de la comédie comme moyen de connaissance de l'homme et de soi, le rapprochent de Molière. Dans une lettre un peu plus tardive à son père, en avril de la même année, il exprime également sa volonté de devenir le Molière allemand. Ici toutefois, la référence à la France reste ambiguë : en revendiquant une forme théâtrale naturelle, dégagée des règles, c'est aussi un rejet du classicisme français qu'il suggère.

Cette confession destinée à sa mère, femme de pasteur à l'instruction limitée, ne se prête pas bien sûr au développement d'une théorie du théâtre mais elle cherche bien à affirmer une identité d'auteur. En 1749, Lessing a déjà six pièces à son actif dont certaines ont été représentées avec grand succès par les Neuber. Il est bien conscient d'avoir trouvé une nouvelle voie dans l'écriture de la comédie. Il s'attaque déjà en ces années à Gottsched, dramaturge et théoricien du théâtre saxon, et à sa comédie satirique (la "Verlachkomödie "), jouée et défendue aussi par la troupe de Neuber. C'est vers la comédie sensible (" die rührende Komödie ") qu'il se tourne dans une perspective qui le rapprochera plus tard de Diderot. Plus encore, des pièces comme Der Freigeist et Die Juden, écrites en 1749 mais publiées seulement en 1754, s'acheminent très perceptiblement vers un théâtre nouveau de l'Aufklärung et préfigurent déjà Nathan der Weise.

Cette lettre contient ainsi une véritable profession de foi, celle d'un intellectuel décidé à en finir avec le conservatisme universitaire et avec ce rigorisme luthérien, incarné par ses parents, qui n'a de cesse de condamner le théâtre et son monde. Lessing a parfaitement pris conscience, déjà à Leipzig puis ensuite à Berlin, que c'est par le journalisme et la scène qu'il pourra faire prévaloir ses idées nouvelles et conquérir un statut d'écrivain indépendant et libre. Au-delà des formules de politesse et des protestations d'obéissance à sa mère, c'est donc comme une déclaration de rupture qu'il faut lire cette lettre. 


\section{À Justina Salomé Lessing}

\section{Très honorée mère,}

Je n'aurais pas tant tardé à prendre la plume si la teneur de ce qu'il me faut vous écrire eût été agréable. Vous devez être vraiment fatiguée de lire des plaintes et des requêtes comme je suis las d'en formuler. Aussi ne croyez pas que vous en trouverez quelque trace dans ces lignes que je vous adresse. Je crains seulement que vous ne me soupçonniez de ne pas être à la hauteur de l'amour et du profond respect dont je vous suis redevable. Je crains seulement que vous n'interprétiez ma présente intervention comme une pure manifestation d'insoumission voire d'une nature mauvaise. Et cette crainte me fâche. Si elle devait s'avérer fondée, cela me peinerait d'autant plus que je me sais innocent. C'est pourquoi permettez-moi de vous dépeindre en quelques traits comment s'est déroulée ma vie universitaire ; vous porterez alors, j'en suis tout à fait certain, un regard plus indulgent sur mon comportement.

Jeune, je quittai l'école fermement convaincu que mon bonheur résidait dans les livres. J'arrivai à Leipzig, une ville où le monde entier se réfléchit comme dans un miroir. J'y vécus les premiers mois dans une retraite plus grande encore que celle que j'avais connue à Meissen. En commerce permanent avec les livres, préoccupé seulement de moi-même, je me souciais aussi peu du reste de l'humanité que - peu s'en faut de Dieu lui-même. Cette confession aurait pour moi un goût quelque peu amer si je n'avais trouvé un motif de consolation, le seul : c'est à mon ardeur au travail, à rien de pire, qu'il faut imputer cette folie. Mais cette situation ne perdura pas, mes yeux se dessillèrent ; je ne saurais dire si c'est pour mon bonheur ou mon malheur : l'avenir en décidera.

Je pris petit à petit conscience que si les livres me rendraient savant, ils ne feraient pas de moi un être humain. J'eus cette audace : je sortis de ma soupente et j'allai à la rencontre de mes contemporains. Dieu du ciel ! Que de différences me séparaient d'eux ! Une timidité de paysan, un corps peu développé et ensauvagé, une totale méconnaissance des usages et des bonnes manières, des airs haïssables qui donnaient à chacun l'impression d'être méprisé, voilà les belles qualités qui me restaient, selon mon propre jugement. Jamais je n'ai éprouvé une telle honte. Elle eut pour effet de me conforter dans la résolution de tout faire pour m'améliorer, et cela, quel qu'en fût le prix. Vous savez vous-même de quelle manière commença cette entreprise. J'appris la danse, l'escrime, l'art équestre. Si mon intention dans cette lettre est de reconnaître mes torts en toute sincérité, elle est aussi de ne pas cacher ce que j'ai fait de bien. Je m'entraînais tant et si bien que je réussis à faire naître une certaine admiration, même chez ceux qui d'avance me déniaient toute adresse en ces domaines.

Ce bon début m'encouragea vivement. Mon corps avait gagné en adresse, et je cherchais la compagnie des hommes pour apprendre aussi ce qu'est la vie. Je mis de côté pour un temps les ouvrages sérieux pour me tourner vers d'autres livres bien plus plaisants, et peut-être tout aussi utiles. Ce sont des comédies qui passèrent tout d'abord entre mes mains. Me croira qui voudra, mais elles me rendirent de bien grands services. Elles m'apprirent à faire la différence entre une pièce de bonne facture et celle qui est corsetée de règles, entre une représentation grossière et celle qui présente un caractère naturel. Elles m'apprirent à distinguer les vraies des fausses vertus, à fuir les vices autant pour leur ridicule que pour leur immoralité. Si je n'ai que faiblement mis en pratique ces leçons, c'est à des circonstances indépendantes de ma volonté qu'il faut l'imputer. Mais pour un peu j'oubliais le plus important profit que je retirai des comédies : j’appris grâce à elles à me connaître moi-même, et depuis lors je peux vous assurer que je n'ai ri et ne me suis moqué de personne autant que de moi-même. Je ne sais pourtant pas comment à cette époque la folle décision de me mettre à écrire moi-même des comédies s'empara de moi. J'osai ! Et quand mes pièces furent représentées à la scène, on voulut bien m'assurer que mon initiative n'avait pas été malheureuse. Il suffit de m'adresser quelque compliment si l'on veut me voir redoubler de sérieux dans l'accomplissement d'un projet. Suite à cela, je réfléchissais nuit et jour à la manière dont je pourrais faire émerger la force d'un talent nouveau dans un genre où aucun Allemand ne s'était encore illustré avec éclat.

Et voilà que soudain, alors que j'étais en plein effort, vous m'enjoignez de regagner le logis familial et jetez le trouble dans mon travail. Ce qui a pu se produire une fois là-bas, vous ne le savez que trop et je ne vais pas vous importuner en me répétant inutilement. On m'accabla particulièrement en m'obligeant à faire connaissance avec certaines personnes que je n'avais croisées que par hasard. Mais je suis redevable à votre bonté d'avoir empêché l'aggravation d'autres 
contrariétés suite, entre autres, aux quelques dettes que j'avais contractées. Je restai tout un trimestre à Kamenz dans une totale inactivité. Dès le début, j'aurais dû dire à quel point j'étais incertain sur mes choix d'études. On me pardonna mes atermoiements jusqu'à un certain moment. Et passé ce délai, veuillez vous souvenir à quel conflit aboutit votre insistance pressante. Je voulais étudier la médecine. Dois-je vous rappeler à quel point vous fûtes alors dépitée ? Uniquement pour vivre selon votre convenance, j'ajoutai alors que j'étais prêt à de longues études et qu’il m'était égal de passer par telle ou telle voie. Fort de cette résolution, je retournai à Leipzig. Mes dettes étaient payées et j'étais convaincu d'être définitivement hors de danger de ce côté. Mais le large cercle de mes connaissances, le mode de vie auquel j'avais habitué mes familiers me précipitèrent à nouveau dans cette ornière. Je pris clairement conscience que si je restais plus longtemps à Leipzig, je ne pourrais sortir de cette impasse inéluctable. La perspective fort déplaisante de vous créer de nouveaux embarras m'incita à prendre la décision de quitter cette ville. Mon choix se porta d'emblée sur Berlin où je pourrais trouver refuge. Par un heureux concours de circonstances, il se trouva qu'à ce moment même Monsieur Lessing ${ }^{1}$ vint de Wittenberg pour me rendre visite. Peu de temps après, je fis avec lui le voyage jusque là-bas avec l'intention de m'y arrêter quelques jours et de faire le tour des lieux; je comptais pouvoir encore gagner Berlin pour l'éclipse de soleil. Mais je tombai malade. Jamais ma propre personne ne me fut autant à charge qu'alors. Pourtant j'y vois là, dans une certaine mesure, la marque de la Providence, si tant est qu'il ne soit pas inconvenant de l'invoquer pour de si petites affaires. Après mon rétablissement, je décidai avec le consentement de Monsieur mon père de passer l'hiver à Wittenberg; et j'espérais bien épargner à nouveau le montant que j’avais investi à Leipzig. Mais je réalisai bientôt que ce que j'avais dépensé pendant ma maladie et en d'autres circonstances, que je préfère taire pour l'instant, dépassait le quart de ma bourse. Mon ancienne résolution d'aller à Berlin me reprit alors. Ce que je fis. Et j'y suis encore. Dans quelles conditions... vous ne le savez que trop.

J'aurais pu depuis longtemps trouver un emploi si j'avais pu faire meilleure impression avec une tenue vestimentaire plus digne. C'est une condition tout à fait nécessaire dans une ville où vous êtes essentiellement jugé sur votre apparence. Il y a de cela une année, vous aviez eu la bonté de me promettre un nouvel habit.
Voyez par vous-même comme cette demande de ma part était fondée. Mais elle fut finalement repoussée sous prétexte que j'étais venu ici à Berlin influencé et attiré par je ne sais qui ${ }^{2}$. Selon toute vraisemblance je devrais encore toucher ma bourse au moins jusqu'à Pâques. Je pourrai ainsi régler à peu près l'ensemble de mes dettes. Cependant je le vois bien : ce jugement infondé que vous portez sur un homme qui ne m'a rendu service que parce que j'en ai eu le plus cruel besoin, ce jugement dénigrant, dis-je, est à l'origine de votre entière opposition à mes entreprises. Cet homme représente-t-il vraiment à vos yeux un rebut de l'humanité ? Ne croyezvous pas que cette haine va trop loin ? Je me console en constatant que je trouve ici, à Berlin, quantité de gens probes et remarquables qui, autant que moi, font grand cas de lui. Voyez pourtant si je lui suis pieds et poings liés comme vous en êtes persuadée. Répondezmoi par une nouvelle lettre ; confirmez-y les propos des précédentes ; conduisez-moi aux mêmes conclusions : aussitôt je quitterai Berlin. Pour autant je ne retournerai pas à la maison. Je ne reviendrai pas non plus à l'université ; le montant de ma bourse ne peut couvrir celui de mes dettes et je n'aurai pas l'impudence de vous infliger de tels frais. Je vais très certainement me rendre à Vienne, Hambourg ou Hanovre ${ }^{3}$. Vous pouvez être assurée que je vous écrirai toujours, où que je sois, et que je n'oublierai jamais les bienfaits que vous m'avez prodigués depuis si longtemps. Dans ces trois villes je peux compter retrouver de bons amis et de solides connaissances. Tout mon parcours m'aura au moins appris une chose : savoir bien me tenir dans le grand monde. Cela me suffira! Je devrais bien finir par trouver un endroit où l'on ait besoin d'un bouche-trou comme moi.

S'il me restait une requête à formuler, ce serait que vous ne doutiez jamais qu'à tout moment je n'ai cessé d'aimer mes parents autant que moi-même. Je ne manquerai pas d'écrire à Monsieur l'inspecteur et au pasteur Lindner ${ }^{4}$, dès que la situation ne fera plus apparaître mes lettres comme une demande réitérée de services. Mon départ de Berlin devrait, je crois, vous apporter l'assurance d'une obéissance pleine et entière à laquelle jamais je ne faillirai tant que je vivrai.

\section{Votre très dévoué fils, Lessing Berlin, le 20 janvier 1749.}

Introduction et traduction : Jean-Louis Elloy 


\section{Notes}

1- Il s'agit de Theophilus Gottlob Lessing, cousin de l'écrivain, qui étudiait alors la théologie à l'université de Wittenberg.

2 - Lessing fait allusion à son ami et mentor Christlob Mylius.

3- En réalité Lessing quittera Berlin seulement en décembre 1751 pour poursuivre ses études de médecine à Wittenberg, mais reviendra à Berlin dès 1752 .

4 - L'« inspecteur " Johann Traugott Lessing et le pasteur Johann Gotthelf Lindner, oncles de l'écrivain, ont souvent soutenu financièrement la famille. 